\title{
17
}

\section{Regular Systems, Ubiquity and Diophantine Approximation}

\author{
V.V. Beresnevich, V.I. Bernik \& M.M. Dodson
}

\begin{abstract}
1 Introduction
Approximation of real and complex numbers by rationals and algebraic numbers appeared first in papers by Dirichlet, Liouville and Hermite on Diophantine approximation and the theory of transcendental numbers. During the first three decades of the 20th century, E. Borel and A. Khintchine introduced the so-called metric (or measure theoretic) approach in which one considers approximation to any number which does not belong to an exceptional null set (i.e., a set of measure zero). Neglecting such exceptional sets can lead to strikingly simple and general theorems, such as Khintchine's theorem (see below). The exceptional sets can be analysed more deeply by using Hausdorff dimension, which can distinguish between different null sets.

This article gives an account of results, methods and ideas connected with Lebesgue measure and Hausdorff dimension of such exceptional sets. We will be concerned mainly with the lower bound of the Hausdorff dimension. Although determining the correct lower bound for the Hausdorff dimension of a set is often (though by no means always) harder than determining the correct upper bound, recent developments indicate that for many problems, the correct lower bound can be established using information associated with the upper bound. There are some exceptions to this principle. For example, convergence in the Khintchine-Groshev type theorem (for terminology see Bernik \& Dodson 1999) for the parabola is related to the upper bound which was proved in Bernik (1979). Nevertheless the divergence case is still unsettled.

For the most part, lower bounds are proved using methods which involve a knowledge of the distribution of some special sets. These sets are very close (or equal) to the solution sets for the Diophantine inequalities under consideration. Originally these methods were developed for sets consisting of points with a distribution described in terms of regular systems. Ubiquitous systems,
\end{abstract}


a multidimensional and more geometrical generalization of regular systems, were introduced in order to investigate more complicated Diophantine approximation, such as on manifolds. Regular and ubiquitous systems have proved to be very effective techniques for obtaining lower bounds for the Hausdorff dimension but in rather different directions.

The development of these ideas has resulted in extensive generalisations of two classical theorems: one due to A.I. Khintchine (see Cassels 1957, Chapter VII, or Khintchine 1924) and the other to V. Jarník (1929) and A.S. Besicovich (1934). Some notation is needed at this point. As usual $|A|$ and $\operatorname{dim} A$ will denote, respectively, the Lebesgue measure and the Hausdorff dimension of the set $A$. Throughout this article, unless otherwise stated, the function $\psi$ : $\mathbb{N} \rightarrow \mathbb{R}^{+}$( $\mathbb{N}$ is the set of positive integers) will be monotonically decreasing. A number $x$ will be called $\psi$-approximable if the inequality

$$
|q x-p|<\psi(q)
$$

holds for infinitely many $(p, q) \in \mathbb{Z} \times \mathbb{N}$. This definition will be carried over to more general and sometimes different situations.

The set of $\psi$-approximable numbers will be denoted by $\mathcal{K}^{1}(\psi)$. Note that $\mathcal{K}^{1}(\psi)$ can be expressed as a general kind of 'lim-sup' set:

$$
\mathcal{K}^{1}(\psi)=\bigcap_{N=1}^{\infty} \bigcup_{q=N}^{\infty} \bigcup_{p \in \mathbb{Z}}\left(\frac{p}{q}-\frac{\psi(q)}{q}, \frac{p}{q}+\frac{\psi(q)}{q}\right) .
$$

The first result shows how the size of $\mathcal{K}^{1}$ in terms of Lebesgue measure depends on the convergence properties of $\psi$.

Theorem 1 (Khintchine) Let $q \psi(q)$ be monotonically decreasing. Then, for any finite interval $I \subset \mathbb{R}$,

$$
\left|\mathcal{K}^{1}(\psi) \cap I\right|=\left\{\begin{aligned}
0, & \text { if } \sum_{q=1}^{\infty} \psi(q)<\infty, \\
|I|, & \text { if } \sum_{q=1}^{\infty} \psi(q)=\infty .
\end{aligned}\right.
$$

The second gives the Hausdorff dimension of the exceptional set $\mathcal{K}_{v}^{1}$ of very well approximable points corresponding to $\psi(q)=q^{-v}$, where $v>1$, in (1).

Theorem 2 (Jarník-Besicovitch) For any $v \geqslant 1$,

$$
\operatorname{dim} \mathcal{K}_{v}^{1}=\frac{2}{v+1} .
$$

The convergence case of Khintchine's theorem and the correct upper bound for the Hausdorff dimension in the Jarník-Besicovitch theorem are quite 
straightforward. Indeed, there are natural covers of the sets $\mathcal{K}^{1}(\psi)$ and $\mathcal{K}_{v}^{1}$ arising from (2) of intervals defined by (1). Applying the Borel-Cantelli Lemma and the Hausdorff-Cantelli Lemma (the Hausdorff dimension analogue of the former, see Bernik \& Dodson 1999, p. 67) to these covers gives the desired results. It is worth repeating that the main difficulty lies in the complementary cases of these theorems.

Regular systems and ubiquitous systems are introduced separately and then some applications are discussed.

\section{Regular systems}

Mahler (1932) gave a classification of real and complex numbers and raised a problem about the approximation type of almost all real numbers. For each $n \in \mathbb{N}$ and $v \in \mathbb{R}$, let $\mathfrak{M}_{v}^{(n)}$ denote the set of $x \in \mathbb{R}$ such that there are infinitely many integer polynomials $P$ of degree at most $n$ satisfying the inequality

$$
|P(x)|<H(P)^{-v},
$$

where $H(P)$ is the height of $P$ (essentially, $\mathfrak{M}_{v}^{(1)}$ is $\mathcal{K}_{v}^{1}$ ). Mahler conjectured that for any $v>n$, the set $\mathfrak{M}_{v}^{(n)}$ is of measure zero. This was solved in V.G. Sprindžuk (1964).

Theorem 3 (Sprindžuk) Let $n \in \mathbb{N}$ and let $v>n$. Then $\left|\mathfrak{M}_{v}^{(n)}\right|=0$.

The Hausdorff dimension of the null set $\mathfrak{M}_{v}^{(n)}$ naturally became of interest. Some upper bounds for $\operatorname{dim} \mathfrak{M}_{v}^{(n)}$ had been obtained before 1964 but no lower bound was known. Baker \& Schmidt (1970) introduced a very powerful method for obtaining lower bounds for Hausdorff dimension and used it to establish the correct lower bound:

$$
\operatorname{dim} \mathfrak{M}_{v}^{(n)} \geqslant \frac{n+1}{v+1} .
$$

Let us now explain the basic ideas of their method. Let $P \in \mathbb{Z}[x], \operatorname{deg} P \leqslant n$ and let $\alpha$ be a real root of $P$. By the continuity of $P$, the closer $x$ is to $\alpha$, the smaller $|P(x)|$. Thus it is very natural to consider approximation of real numbers by real algebraic numbers in (1) with $\psi(q)=q^{-v}$.

Let $\mathbb{A}^{(n)}$ denote the set of real algebraic numbers of degree at most $n$. Given $v \in \mathbb{R}$, let $\mathbb{A}_{v}^{(n)}$ be the set of $x \in \mathbb{R}$ such that there are infinitely many $\alpha \in \mathbb{A}^{(n)}$ satisfying

$$
|x-\alpha|<H(\alpha)^{-v-1}
$$


where $H(\alpha)$ is the height of $\alpha$. It is not difficult to see that if $n<w<v$, then

$$
\mathbb{A}_{v}^{(n)} \subset \mathfrak{M}_{w}^{(n)} .
$$

Thus, since $A \subset B$ implies that $\operatorname{dim} A \leqslant \operatorname{dim} B$, a lower bound for $\operatorname{dim} \mathbb{A}_{v}^{(n)}$ is also a lower bound for $\operatorname{dim} \mathfrak{M}_{w}^{(n)}$. Note that the inequality $\operatorname{dim} \mathbb{A}_{v}^{(n)} \leqslant(n+$ $1) /(v+1)$ can be easily obtained by the Hausdorff-Cantelli Lemma exactly as in the case $n=1$, which has already been discussed.

Now let us consider the rationals again. They are dense in $\mathbb{R}$ and also uniformly distributed. Moreover, in a certain sense any two different rational numbers are well separated. This can be described as follows. Let $T$ be a large positive number. Let $\mathbb{Q}_{T}$ be the set of rationals with height (the modulus of the denominator) less than or equal to $T$. The number of such rationals in an interval $I, \operatorname{card}\left(\mathbb{Q}_{T} \cap I\right)$, is $O\left(T^{2}|I|\right)$. It is easily seen that the average of the distances between two consecutive rationals in $\mathbb{Q}_{T} \cap I$ is asymptotically $|I| / \operatorname{card}\left(\mathbb{Q}_{T} \cap I\right)=O\left(T^{-2}\right)$. Also the distance between two consecutive rationals in $\mathbb{Q}_{T} \cap I$ is at least $T^{-2}$. Thus on average the points of $\mathbb{Q}_{T} \cap I$ are separated as they are individually.

This is not the case for algebraic numbers of higher degree. However, it can be shown that a positive proportion of points in $\mathbb{A}^{(n)}(T)$, the set of algebraic numbers of degree at most $n$ and height at most $T$, consists of well separated points. Thus the set $\mathbb{A}^{(n)}(T)$ can be refined so that we will have a system of points with a distribution similar to the rationals. This fact, first established by Baker \& Schmidt (1970), is described using the concept of a regular system of points.

Definition 1 Let $\Gamma$ be a countable set of real numbers and let $N: \Gamma \rightarrow \mathbb{R}$ be a positive function. The pair $(\Gamma, N)$ is called a regular system of points if there exists a constant $C_{1}=C_{1}(\Gamma, N)>0$ such that for any finite interval $I$ there exists a sufficiently large number $T_{0}=T_{0}(\Gamma, N, I)>0$ such that for any integer $T \geqslant T_{0}$ there exists a collection

$$
\gamma_{1}, \ldots, \gamma_{t} \in \Gamma \cap I
$$

such that $N\left(\gamma_{i}\right) \leqslant T \quad(1 \leqslant i \leqslant t),\left|\gamma_{i}-\gamma_{j}\right| \geqslant T^{-1} \quad(1 \leqslant i<j \leqslant t)$, and $t \geqslant C_{1}|I| T$.

Example 2 It is readily verified that the set of all rational numbers together with the function $N(p / q)=q^{2}$, where $p$ and $q$ are relatively prime, is a regular system. 
As usual, $\{x\}$ denotes the fractional part of the real number $x$ and $\|x\|=$ $\min \{|x-k|: k \in \mathbb{Z}\}$. A number $\alpha$ is badly approximable if $\inf \{n\|n \alpha\|: n \in$ $\mathbb{N}\}>0$.

Example 3 When $\alpha \in \mathbb{R}$ is a badly approximable number, the pair $(\Gamma, N)$, where $\Gamma=\{\{\alpha n\}: n \in \mathbb{N}\}$ and $N(\{\alpha n\})=n$, is a regular system.

The following non-trivial example of a regular system was given by Baker $\&$ Schmidt (1970).

Example 4 Let $\Gamma=\mathbb{A}^{(n)}$ and $N(\gamma)=H(\gamma)^{n+1}(\log H(\gamma))^{-3 n(n+1)}$ for $\gamma \in$ $\Gamma$. Then $(\Gamma, N)$ is a regular system.

The next lemma is the key point of the Baker-Schmidt method for obtaining lower bounds for Hausdorff dimension (see Baker \& Schmidt 1970, Rynne 1992).

Lemma 1 Suppose that $\psi: \mathbb{R} \rightarrow \mathbb{R}^{+}$is decreasing with $x \psi(x) \leqslant 1 / 2$ for large $x$. If $(\Gamma, N)$ is a regular system then

$$
\operatorname{dim} \Lambda(\Gamma, N ; \psi) \geqslant s_{0}=\sup \left\{s: \lim _{x \rightarrow \infty} x \psi(x)^{s}=\infty\right\},
$$

where $\Lambda(\Gamma, N, \psi)$ is the set of all real numbers $x$ for which the inequality $|x-\gamma|<\psi(N(\gamma))$ holds for infinitely many $\gamma \in \Gamma$.

Baker \& Schmidt (1970) applied this lemma to Example 4 and used inequality (8) to establish the correct lower bounds for $\operatorname{dim} \mathbb{A}_{v}^{(n)}$ and $\operatorname{dim} \mathfrak{M}_{v}^{(n)}$. The correct upper bound for $\operatorname{dim} \mathbb{A}_{v}^{(n)}$ can be easily obtained by Hausdorff-Cantelli Lemma in the same way as in the case $n=1$ already discussed. Determining the correct upper bound for $\operatorname{dim} \mathfrak{M}_{v}^{(n)}$ is much harder and involves different arguments based on careful and complicated analysis of the distribution of all the algebraic numbers, not only regularly distributed ones, since any subclass of $\mathbb{A}_{v}^{(n)}$ may contribute to $\operatorname{dim} \mathfrak{M}_{v}^{(n)}$ (Bernik 1983).

Theorem 4 For any $v>n$,

$$
\operatorname{dim} \mathbb{A}_{v}^{(n)}=\operatorname{dim} \mathfrak{M}_{v}^{(n)}=(n+1) /(v+1) .
$$

Melián \& Pestana (1993) have considered the hyperbolic space analogue of the Jarník-Besicovitch theorem. To obtain the correct lower bound for the Hausdorff dimension, they used 'well-distributed' systems, an extension of regular systems to higher dimensions. 


\section{Ubiquity}

Ubiquitous systems were introduced in Dodson, Rynne \& Vickers (1990) as another technique for obtaining a lower bound for the Hausdorff dimension of sets of 'very well approximable' points and of general 'exceptional' sets associated with questions of 'small denominators' which arise in normal forms and stability of dynamical systems (see Arnold 1963, Bernik \& Dodson 1999, Chapter 7, and Dodson, Rynne \& Vickers 1989, for more details). In one dimension, ubiquitous and regular systems, discussed above, are almost equivalent (regular systems are more delicate in one respect but ubiquity has been extended to include this in Rynne 1992). Regular systems lend themselves to more refined simultaneous estimates in higher dimensions but ubiquitous systems deal with broader questions and yield a lower bound for the Hausdorff dimension more directly in terms of the geometry. In addition, ubiquity allows the approximation function $q^{-v}$ to be replaced naturally by $\psi: \mathbb{N} \rightarrow \mathbb{R}^{+}$, where $\psi(q) \rightarrow 0$ monotonically as $q \rightarrow \infty$.

In the type of Diophantine approximation considered here, we are concerned with the set consisting of points $x$ in Euclidean space which are, roughly speaking, a small distance from a member of a special class of subsets of the space infinitely often. The set is related to a general sort of 'lim-sup' of a sequence of neighbourhoods of special sets. In the Jarník-Besicovitch theorem described above, the special class of subsets is the set of rationals $\mathbb{Q}$ and the distance is less than $q^{-v}$. There is no loss of generality in confining attention to the (open or closed) unit interval or to hypercubes in higher dimensions. The hard part of this theorem is establishing the correct lower bound for $\operatorname{dim} \mathcal{K}_{v}^{1}$. As has been pointed out, this can be obtained using regular systems, which were introduced to establish the generalisation of the Jarník-Besicovitch theorem to approximation by real algebraic irrationals of given degree (Baker \& Schmidt 1970). Ubiquity, however, was framed to deal with higher dimensional sets, such as the systems of linear forms arising in a general form of the Jarník-Besicovitch theorem established by Bovey \& Dodson (1986). For each $\mathbf{x}=\left(x_{1}, \ldots, x_{n}\right) \in \mathbb{R}^{n}$, we let

$$
|\mathbf{x}|=\max \left\{\left|x_{1}\right|, \ldots,\left|x_{n}\right|\right\} \text { and }\|\mathbf{x}\|=\max \left\{\left\|x_{1}\right\|, \ldots,\left\|x_{n}\right\|\right\}
$$

be the height of $\mathbf{x}$ and the distance of $\mathbf{x}$ from $\mathbb{Z}^{n}$ respectively. Let $\mathcal{K}^{m, n}(\psi)$ be the set of real $m \times n$ matrices $\left(a_{i j}\right)=A$, regarded as points in $\mathbb{R}^{m n}$, such that the system of inequalities

$$
\|\mathbf{q} A\|=\max _{j=1, \ldots, n}\left\{\left\|q_{1} a_{1 j}+\ldots+q_{m} a_{m j}\right\|\right\}<\psi(|\mathbf{q}|)
$$

holds for infinitely many $\mathbf{q}=\left(q_{1}, \ldots, q_{m}\right) \in \mathbb{Z}^{m}$. The set $\mathcal{K}^{m, n}(\psi)$ is related 
to a 'lim-sup' set of a sequence of neighbourhoods of finite unions of subsets of hyperplanes $R(\mathbf{q})=\left\{A \in(0,1)^{m n}:\|\mathbf{q} A\|=0\right\}$. For another more complicated example, see Dodson, Rynne \& Vickers (1994). Following Arnol'd (1983), these sets $R(\mathbf{q})$ are called resonant because of the association with the physical phenomenon of resonance.

The definition of ubiquity was abstracted from Bovey \& Dodson (1986) which involved systems of linear forms and used geometrical ideas based on those in Besicovitch (1934) combined with a mean and variance argument from Cassels (1957), Chapter 7. By design, resonant sets play a fundamental role in ubiquity which in essence ensures that they are in good supply. They can be thought of as generalisations of rational numbers and are of a relatively simple nature, being finite unions of points or parts of lines, curves, planes, surfaces and so on, which are solution sets of Diophantine equations.

Definition 2 Take $U$ to be a non-empty open subset of $\mathbb{R}^{m}$. Let

$$
\mathcal{R}=\left\{R_{j} \subset U: j \in J\right\}
$$

be a family of resonant sets, indexed by $J$, where each $j \in J$ has a weight $\lfloor j\rfloor>0$. The resonant set $R_{j}=R(\mathbf{q})$ with $\lfloor j\rfloor=|\mathbf{q}|$ in the above. Let the function $\rho: \mathbb{N} \rightarrow \mathbb{R}^{+}$converge to 0 at $\infty$ and let $A(Q), Q=1,2, \ldots$, be a sequence of subsets of $U$ such that $\lim _{Q \rightarrow \infty}|A(Q)|=|U|$. Let

$$
B\left(R_{j} ; \delta\right)=\left\{u \in U: \operatorname{dist}_{\infty}\left(u, R_{j}\right)<\delta\right\},
$$

where $\operatorname{dist}_{\infty}(u, R)=\inf \{|u-r|: r \in R\}$, the distance from $u$ to $R$ in the supremum norm. Suppose that there exists a constant $d \in[0, m]$ such that given any hypercube $H \subset U$ with $\ell(H)=\rho(Q)$ and such that $H / 2$ intersects $A(Q)$, then there exists a $j \in J$ with $\lfloor j\rfloor \leqslant Q$ such that for all $\delta \in(0, \rho(Q)]$,

$$
\left|H \cap B\left(R_{j} ; \delta\right)\right| \gg \delta^{m-d} \ell(H)^{d},
$$

where $\gg$ and $\ll$ are the Vinogradov symbols $(b \gg a$ and $a \ll b$ mean that $a=$ $O(b)$ ). Suppose further that for any other hypercube $H^{\prime}$ in $U$ with $\ell\left(H^{\prime}\right) \leqslant$ $\rho(Q)$,

$$
\left|H^{\prime} \cap H \cap B\left(R_{j} ; \delta\right)\right| \ll \delta^{m-d} \ell\left(H^{\prime}\right)^{d} .
$$

Then the pair $(\mathcal{R}, L \cdot\rfloor)$ is called a ubiquitous system with respect to $\rho$ (reference to the weight is usually omitted).

The intersection estimates (11) and (12) have been used in preference to more geometrical descriptions of the intersections $H \cap R_{j}$ for generality. The 
first requires that the hypercube $H$ and the resonant set $R_{j}$ intersect substantially and that small hypercubes $H^{\prime}$ intersect $H \cap R_{j}$ as they 'should'. For resonant sets $R_{j}$ with a reasonable structure, $d$ will be the topological dimension of each $R_{j}$ and the intersection conditions (11) and (12) will be satisfied more or less automatically. Indeed when the $R_{j}$ are $d$-dimensional affine spaces in Euclidean space, we can take the approximating set $A(Q)$ to be a union of $\rho(Q)$-neighbourhoods of $R_{j}$, namely $A(Q)=\bigcup_{\lfloor j\rfloor \leqslant Q} B\left(R_{j} ; \rho(Q)\right)$. It is then readily verified that the intersection conditions (11) and (12) can be replaced by the single measure condition

$$
\left|\bigcup_{\lfloor j\rfloor \leqslant Q} B\left(R_{j} ; \rho(Q)\right)\right| \rightarrow|U| \text { as } Q \rightarrow \infty .
$$

This condition can be weakened to the left-hand side being at least $c|U|$ for some constant $c, 0<c \leqslant 1$ and all $Q$ sufficiently large, see Rynne (1992). Ubiquity can be relatively simple to establish and in practice the function $\rho$ emerges naturally. For instance Dirichlet's theorem implies that the set of rationals (with weight the modulus of the denominator) is ubiquitous with respect to a function comparable with $Q^{-2} \log Q ;$ in $\mathbb{R}^{n}$, the rational points $\mathbf{p} / q$, where $\mathbf{p} \in \mathbb{Z}^{n}, q \in \mathbb{Q}$, are ubiquitous with respect to a function comparable with $Q^{-1-1 / n} \log Q$, see Dodson, Rynne \& Vickers (1990).

\section{A general lower bound}

The distribution of the resonant sets in ubiquitous systems allows the determination of a general lower bound for the Hausdorff dimension of the lim-sup set

$$
\Lambda(\mathcal{R} ; \psi)=\left\{u \in U: \operatorname{dist}_{\infty}\left(u, R_{j}\right)<\psi(\lfloor j\rfloor) \text { for infinitely many } j \in J\right\}
$$

where $\psi: \mathbb{N} \rightarrow \mathbb{R}^{+}$is a non-increasing function and the resonant sets have common dimension $d=\operatorname{dim} \mathcal{R}$, say, and codimension $m-d=\operatorname{codim} \mathcal{R}$.

Theorem 5 Suppose $\mathcal{R}$ is a family of resonant sets which is ubiquitous with respect to $\rho$ and that $\widetilde{\psi}: \mathbb{R}^{+} \rightarrow \mathbb{R}^{+}$is a non-increasing function satisfying $\widetilde{\psi}(Q) \leqslant \rho(Q)$ for $Q$ sufficiently large. Then

$$
\operatorname{dim} \Lambda(\mathcal{R} ; \tilde{\psi}) \geqslant \operatorname{dim} \mathcal{R}+\gamma \operatorname{codim} \mathcal{R},
$$

where $\gamma=\lim \sup _{Q \rightarrow \infty} \log \rho(Q) / \log \widetilde{\psi}(Q) \leqslant 1$. 
This is proved in Dodson, Rynne \& Vickers (1990) and another proof using the mass distribution principle is given in Bernik \& Dodson (1999), Chapter 5. The constant $\gamma$ is at most 1 since $\widetilde{\psi}(Q) \leqslant \rho(Q)$ for $Q$ sufficiently large.

This result can be used to establish the correct lower bound for the Hausdorff dimension of $\psi$-approximable systems of linear forms. It can be shown using Minkowski's linear forms theorem that the resonant sets $R_{\mathbf{q}}$, where $\mathbf{q} \in \mathbb{Z}^{m}$ is non-zero, given by

$$
R_{\mathbf{q}}=\left\{A \in[0,1]^{m n}: \mathbf{q} A \in \mathbb{Z}^{n}\right\}
$$

are ubiquitous with respect $m Q^{-1-m / n} \log Q$ and 'most' matrices $A$ in $[0,1]^{m n}$ are 'close' to a set $R_{\mathbf{q}}$ with weight $\lfloor\mathbf{q}\rfloor=|\mathbf{q}|$ not too large (see Dodson 1992, 1993). The correct upper bound for the Hausdorff dimension of $\mathcal{K}^{m, n}(\psi)$ can obtained using a straightforward covering argument. The complementary result follows from Theorem 5 with $\widetilde{\psi}(Q) \asymp Q \psi(Q)(a \asymp b$ means that $a$ and $b$ are comparable, i.e., $a \ll b$ and $b \ll a)$. The lower order $\lambda(f)$ of a function $f: \mathbb{N} \rightarrow \mathbb{R}^{+}$is defined to be $\liminf _{Q \rightarrow \infty}(\log f(Q)) /(\log Q)$.

Theorem 6 Let $\psi: \mathbb{N} \rightarrow \mathbb{R}^{+}$be a decreasing function and let $\lambda$ be the lower order of $1 / \psi$. Then

$$
\operatorname{dim} \mathcal{K}^{m, n}(\psi)= \begin{cases}(m-1) n+(m+n) /(\lambda+1) & \text { when } \lambda \geqslant m / n \\ m n & \text { when } \lambda \leqslant m / n\end{cases}
$$

The Jarník-Besicovitch theorem corresponds to $m=n=1$ and $\psi(q)=q^{-v}$. Dickinson \& Velani (1997) have extended Jarník's Hausdorff measure analogue (Jarník 1929) of Khintchine's theorem for simultaneous Diophantine approximation to systems of linear forms, thus establishing the Hausdorff measure analogue of the Khintchine-Groshev theorem (Sprindžuk 1979). Instead of ubiquity, they work with an elaborate Cantor-type construction. There are some interesting applications to normal forms of pseudodifferential operators (Dickinson, Gramchev, \& Yoshino 1995). The complete hyperbolic analogue of the Jarník-Besicovitch theorem was established by Hill \& Velani (1998) using Cantor type subsets.

Inhomogeneous Diophantine approximation, which in one dimension concerns the size of $|q x-\alpha-p|$ for some fixed $\alpha \in \mathbb{R}$, differs somewhat from homogeneous Diophantine approximation, where $\alpha=0$. It is easier in the doubly metric case where one considers the joint measure of the set of points $(x, \alpha)$ but harder in the singly metric case where $\alpha$ is given. Using ubiquity, a general form of the inhomogeneous version of the Jarník-Besicovitch theorem has been obtained for the doubly metric case (Dodson 1997) and Levesley 
(1998) has established it in the more difficult singly metric case with the additional help of uniform distribution.

Dickinson has discussed the Hausdorff dimension for systems of linear forms which have small modulus and has shown that the set of matrices $A=\left(a_{i j}\right) \in \mathbb{R}^{m n}$ such that for infinitely many $\mathbf{q} \in \mathbb{Z}^{m}$,

$$
|\mathbf{q} A|=\max _{j=1, \ldots, n}\left\{\left|q_{1} a_{1 j}+\ldots+q_{m} a_{m j}\right|\right\}<|\mathbf{q}|^{-v}
$$

has Hausdorff dimension $m(n-1)+m /(v+1)$ for $v \geqslant(m / n)-1$ and $m n$ otherwise (Dickinson 1993). The upper bound is obtained by the usual covering argument but ubiquity gives the correct lower only for $m>n$. In the complementary range where $m \leqslant n$, a diffeomorph of the set is decomposed into the cartesian product of an $(m-1)(n-m+1)$-cube and a space to which the arguments in the first range apply. For a more general approach, see Dickinson (1993) and Rynne (1998a,b). The $p$-adic version of the general JarníkBesicovitch theorem is essentially of the form (13). The Hausdorff dimension of the corresponding set was obtained by Abercrombie (1995) for $m>n$ using Billingsley dimension; the dimension when $m \leqslant n$ has been determined in Dickinson, Dodson \& Jin (1999) using the same approach as in Dickinson (1993).

\section{Khintchine-type theorems on manifolds}

Theorem 1 has been generalised to approximation by real algebraic numbers and to Diophantine approximation on manifolds in Euclidean space. The functional dependence between the coordinates in the latter case causes formidable technical problems but approximation on the rational normal curve

$$
\mathcal{V}=\left\{\left(x, \ldots, x^{n}\right): x \in \mathbb{R}\right\}
$$

is related to approximation by real algebraic numbers. In this connection, in 1966 Baker raised (with a slightly different notation) the question of the measure of the set $\mathfrak{M}^{(n)}(\psi)$ of $x \in \mathbb{R}$ such that the inequality

$$
|P(x)|<\psi(H(P))
$$

has infinitely many solutions $P \in \mathbb{Z}[x]$ with $\operatorname{deg} P \leqslant n$. Baker proved that $\left|\mathfrak{M}^{(n)}(\psi)\right|=0$ if $\psi$ is monotonic and if $\sum_{q=1}^{\infty} \psi^{1 / n}(q)<\infty$ (see Baker 1966). He further conjectured that the convergence condition can be replaced with $\sum_{q=1}^{\infty} q^{n-1} \psi(q)<\infty$; this was proved by Bernik (1989), see also Bernik \& Dodson (1999). 
Theorem 7 Let $\psi: \mathbb{N} \rightarrow \mathbb{R}^{+}$be monotonic. Then $\left|\mathfrak{M}^{(n)}(\psi)\right|=0$ whenever the sum

$$
\sum_{q=1}^{\infty} q^{n-1} \psi(q)
$$

converges.

The proof of this result was used to improve the regular system of algebraic numbers constructed by Baker \& Schmidt.

Example 5 (see Bernik \& Dodson 1999, p. 101). For each $n \in \mathbb{N}$, let

$$
N(\gamma)=H(\gamma)^{n+1}(\log H(\gamma))^{-(n+1)}
$$

for $\gamma \in \mathbb{A}^{(n)}$. Then $\left(\mathbb{A}^{(n)}, N\right)$ is a regular system.

Regular systems were used to establish a Khintchine-Groshev type theorem for $\mathfrak{M}^{(n)}(\psi)$ when the sum (16) diverges (Beresnevich 1999):

Theorem 8 Let $\psi: \mathbb{N} \rightarrow \mathbb{R}^{+}$be a monotonic sequence. Then for each $n \in \mathbb{N}$, the set $\mathfrak{M}^{(n)}(\psi)$ has full measure $\dagger$ whenever $\sum_{q=1}^{\infty} q^{n-1} \psi(q)=\infty$.

Theorem 8 can be derived from Theorem 9 below using the following arguments. Let $\mathbb{A}^{(n)}(\psi)$ be the set consisting of $x \in \mathbb{R}$ such that there are infinitely many $\gamma \in \mathbb{A}^{(n)}$ satisfying

$$
|x-\gamma|<\psi(H(\gamma))
$$

It can be verified easily that for any interval $I_{0}$, there is a sufficiently small positive constant $c$ such that $\mathbb{A}^{(n)}(\tilde{\psi}) \cap I_{0} \subset \mathfrak{M}^{(n)}(\psi) \cap I_{0}$ if $\tilde{\psi}(q) \leqslant c \psi(q) / q$ for all $q \in \mathbb{N}$. Thus the following suffices to prove Theorem 8 , see Beresnevich (1999).

Theorem 9 For any $n \in \mathbb{N}$ and monotonic sequence $\psi: \mathbb{N} \rightarrow \mathbb{R}^{+}$, the set $\mathbb{A}^{(n)}(\psi)$ has full measure whenever $\sum_{q=1}^{\infty} q^{n} \psi(q)=\infty$.

Regular systems play the key role in the proof of Theorem 9. Indeed this Khintchine-type result requires 'best possible' knowledge about the distribution of real algebraic numbers. Given an interval $I$ and a positive number $T$, the collection (9) is chosen from the set $\Gamma_{N}(I, T)=\{\gamma \in \Gamma \cap I: N(\gamma) \leqslant T\}$.

$\dagger$ A set $A$ has full measure if $|\mathbb{R} \backslash A|=0$ 
This set may contain many points which are not included in (9). For example, in the Baker-Schmidt regular system (see Example 4),

$$
\frac{\operatorname{card} \Gamma_{N}(I, T)}{\text { the number of points satisfying (9) }} \asymp|I|(\log T)^{3 n(n+1)} \text {. }
$$

In Example 5 this ratio is $\asymp|I|(\log T)^{n+1}$, a little smaller. This suggests the following.

Definition 3 (see Beresnevich 2000.) The regular system $(\Gamma, N)$ will be called best possible if for any finite interval $I$

$$
\sup _{T>0} \frac{\operatorname{card} \Gamma_{N}(I, T)}{T}<\infty
$$

The following example of a best possible regular system is given in Beresnevich (1999) where more details are given.

Example 6 For each $n \in \mathbb{N}$, let $N(\gamma)=H(\gamma)^{n+1} /(1+|\gamma|)^{n(n+1)}$. Then $\left(\mathbb{A}^{(n)}, N\right)$ Then $(\Gamma, N)$ is a regular system.

The proof of this example is based on measuring the solution sets for certain Diophantine inequalities efficiently (see Beresnevich 1999 for more details). The proof of Theorem 9 is based on the following generalised BorelCantelli lemma, also used in the proof of the Khintchine-Groshev theorem (see Sprindžuk 1979, Chapter 2,§ 2; Harman 1998, p. 35).

Lemma 2 Let $E_{i} \subset \mathbb{R}$ be a sequence of measurable sets and the set $E$ consist of points $x$ belonging to infinitely many $E_{i}$. If all the sets $E_{i}$ are uniformly bounded and the sum $\sum_{i=1}^{\infty}\left|E_{i}\right|$ diverges, then

$$
|E| \geqslant \limsup _{N \rightarrow \infty} \frac{\left(\sum_{i=1}^{N}\left|E_{i}\right|\right)^{2}}{\sum_{i=1}^{N} \sum_{j=1}^{N}\left|E_{i} \cap E_{j}\right|} .
$$

The sets $E_{i}$ are taken to be small neighbourhoods of points (9). The set $\mathbb{A}^{(n)}$ having a best possible regular system makes it possible to control the sum in both the numerator and the denominator of (19). As far as approximation by points of regular system is concerned, the following Khintchine-type result is proved in Beresnevich (2000). 
Lemma 3 Let $(\Gamma, N)$ be a regular system, $\psi: \mathbb{N} \rightarrow \mathbb{R}^{+}$be monotonic and $\Lambda(\Gamma, N ; \psi)$ be the set defined in Lemma 1. Then, for any interval $I \subset \mathbb{R}$

$|\Lambda(\Gamma, N ; \psi) \cap I|=\left\{\begin{aligned} 0, & \text { if } \sum_{q=1}^{\infty} \psi(q)<\infty \text { and }(\Gamma, N) \text { is best possible, } \\ |I|, & \text { if } \sum_{q=1}^{\infty} \psi(q)=\infty .\end{aligned}\right.$

Extending Khintchine-type theorems to a manifold $M$ in $\mathbb{R}^{n}$ is difficult because of the functional relationships between the coordinates of points (the measure is of course the induced Lebesgue measure on $M$ ). A half-way house is to show that the set $\mathcal{L}(M ; \psi)$ of points $\mathbf{x} \in M$ such that

$$
\|\mathbf{x} \cdot \mathbf{q}\|<\psi(|\mathbf{q}|)
$$

for infinitely many $\mathbf{q} \in \mathbb{Z}^{n}$ is null (in the induced measure on $M$ ) when $\psi(q)=$ $q^{-v}$ for any $v>n$. The set $\mathcal{L}(M ; \psi)$ is dual to the set $\mathcal{S}(M ; \psi)$ of points $\mathbf{x} \in M$ satisfying

$$
\max \left\{\left\|q x_{i}\right\|: i=1, \ldots, n\right\}<\psi(q)
$$

for infinitely many $q \in \mathbb{N}$. By Khintchine's Transference Principle, the set $\mathcal{S}_{v}(M)$, which is $\mathcal{S}(M ; \psi)$ with $\psi(q)=q^{-v}$, is also null for $v>1 / n$ (see Bernik \& Dodson 1999).

The first general result in the metrical theory of Diophantine approximation on manifolds was due to Schmidt (1964). He investigated $C^{3}$ planar curves of the form $\Gamma=\left\{\left(f_{1}(x), f_{2}(x)\right): x \in I\right\}$, where $I$ is an interval, $f_{1}, f_{2}: I \rightarrow \mathbb{R}$ are $C^{3}$ functions such that the curvature $f_{1}^{\prime}(s) f_{2}^{\prime \prime}(s)-f_{1}^{\prime \prime}(s) f_{2}^{\prime}(s) \neq 0$ for almost all $s \in I$, and proved that for any such curve, the set of very well approximable points is relatively null, i.e., the curve is extremal (see Bernik \& Dodson 1999 for terminology). Thus $M$ is extremal if the set of simultaneously very well approximable points

$$
\mathcal{S}_{v}(M)=\left\{\xi \in M:\|q \xi\|<q^{-v} \text { for infinitely many } q \in \mathbb{N}\right\}
$$

is relatively null when $v>1 / n$ or equivalently if the set

$$
\mathcal{L}_{v}(M)=\left\{\xi \in M:\|\mathbf{q} \cdot \xi\|<|\mathbf{q}|^{-v} \text { for infinitely many } \mathbf{q} \in \mathbb{Z}^{n}\right\}
$$

is null in $M$ when $v>n$ respectively. The terminology reflects the fact that for almost all points on an extremal set, the exponents in Dirichlet's theorem are unimprovable, for more details see Bernik \& Dodson (1999), Koksma (1936) p. 67. A manifold $M$ is strongly extremal if given any $v>n$, the set of points $x=\left(x_{1}, \ldots, x_{n}\right) \in M$ satisfying

$$
\|\mathbf{q} \cdot \mathbf{x}\|<\prod_{j=1}^{n}\left(\left|q_{j}\right|+1\right)^{-v / n}
$$


for infinitely many $\mathbf{q} \in \mathbb{Z}^{n}$ is null in $M$, so that a strongly extremal manifold is extremal. Baker conjectured that the rational normal curve $\mathcal{V}$ (see (14)) is strongly extremal in Baker (1975) and Sprindžuk extended the conjecture to any manifold $M$ satisfying the conditions of $\mathrm{H}_{1}$ (Conjecture $\mathrm{H}_{2}$ in Sprindžuk 1980). The rational normal curve $\mathcal{V}$ was shown to be strongly extremal by Bernik \& Borbat (1997) for $n=4$.

Manifolds satisfying a variety of analytic, geometric and number theoretic conditions have been shown to be extremal (more details are in Bernik \& Dodson 1999; Sprindžuk 1979, 1980; Vinogradov \& Chudnovsky 1984). In an extension of Schmidt's theorem to higher dimensional manifolds, Kovalevskaya (1978) has shown that surfaces in $\mathbb{R}^{3}$ having non-zero Gaussian curvature almost everywhere are extremal (see also or Sprindžuk 1979, p. 149, Theorem 18) and, together with Bernik, later extended this result to $m$-dimensional surfaces in $\mathbb{R}^{2 m}$, see Bernik \& Kovalevskaya (1990). These are special cases of the more general result that smooth $\left(C^{3}\right)$ manifolds of dimension at least 2 (so that $M$ is at least a surface) and satisfying a curvature condition (which specialises to non-zero Gaussian curvature for surfaces in $\mathbb{R}^{3}$ ) are also extremal (see Dodson, Rynne \& Vickers 1989, 1991, and the next section).

Schmidt's result has been extended to $C^{4}$ curves in $\mathbb{R}^{3}$ by Beresnevich $\&$ Bernik (1996). Recently Kleinbock \& Margulis (1998) have proved that manifolds which are nondegenerate almost everywhere are strongly extremal. Nondegeneracy can be regarded as a generalisation of non-zero curvature and is defined as follows. For each $j \leqslant k$, the point $\mathbf{x}=\theta(u) \in M \subset \mathbb{R}^{n}$ is $j$ nondegenerate if the partial derivatives of $\theta$ at $u$ up to order $j$ span $\mathbb{R}^{n}$. The point $\mathbf{x}$ is nondegenerate if it is $j$-nondegenerate for some $j$. This result is best possible and implies both Sprindžuk's and the stronger Baker-Sprindžuk conjectures. The proof uses ideas from dynamical systems, particularly unipotent flows in homogeneous spaces of lattices. Their techniques are likely to lead to further progress and have led to a generalisation of Baker's result (Baker 1966).

In 1991, the following Khintchine-Groshev-type result was obtained for fairly general manifolds. Let $M$ be a $C^{3}$ manifold embedded in $\mathbb{R}^{n}$ with dimension at least 2 and 2-convex almost everywhere (i.e., $M$ has at least 2 principal curvatures with strictly positive product almost everywhere). Then $\mathcal{L}(M ; \psi)$ is null if the sum (16) converges. If the sum diverges and $M$ satisfies a stronger curvature condition, then $\mathcal{L}(M ; \psi)$ is full (Dodson, Rynne \& Vickers 1991, Theorem 1.1. If the sum $\sum_{q=1}^{\infty} \psi(q)^{n}$ converges, then $\mathcal{S}(M ; \psi)$ is null. Khintchine-Groshev type analogues of Schmidt's theorem were obtained in Beresnevich, Bernik, Dodson \& Dickinson (1999) and Bernik, Dodson \& 
Dickinson (1998). A slightly different notation has been adopted, reflecting the connection with $\mathfrak{M}^{(n)}(\psi)$.

Theorem 10 Let $I$ be a finite interval and $f_{1}, f_{2}: I \rightarrow \mathbb{R}$ be $C^{3}$ functions such that $f_{1}^{\prime}(x) f_{2}^{\prime \prime}(x)-f_{1}^{\prime \prime}(x) f_{2}^{\prime}(x) \neq 0$ for almost all $x \in I$. Let $\psi: \mathbb{N} \rightarrow \mathbb{R}^{+}$be monotonic and let $\mathcal{L}_{f_{1}, f_{2}}(\psi)$ be the set of $x \in I$ such that the inequality

$$
\left|a_{2} f_{2}(x)+a_{1} f_{1}(x)+a_{0}\right|<\psi\left(|\mathbf{a}|_{\infty}\right),
$$

where $|\mathbf{a}|_{\infty}=\max \left\{\left|a_{0}\right|,\left|a_{1}\right|,\left|a_{2}\right|\right\}$, has infinitely many solutions $\mathbf{a}=$ $\left(a_{0}, a_{1}, a_{2}\right) \in \mathbb{Z}^{3}$. Then

$$
\left|\mathcal{L}_{f_{1}, f_{2}}(\psi)\right|=\left\{\begin{aligned}
0, & \text { if } \sum_{q=1}^{\infty} q \psi(q)<\infty \\
|I|, & \text { if } \sum_{q=1}^{\infty} q \psi(q)=\infty
\end{aligned}\right.
$$

Analogues of Khintchine's theorem have been obtained recently for nondegenerate manifolds. This was done independently by Beresnevich (2001b) and Bernik, Kleinbock \& Margulis (1999). In the former, a development of Sprindžuk's method of essential and inessential domains is applied to smooth curves with Wronskians which are non-zero almost everywhere and the result extended to nondegenerate manifolds. In the latter, the geometry of lattices in Euclidean spaces and flows on homogeneous spaces are used $\dagger$. The complementary divergence case has also been established for various cases in Beresnevich (1999, 2000, 2001a, 2001b) and Bernik, Kleinbock \& Margulis (1999), and the full result seems likely to be proved soon.

\section{Hausdorff dimension on manifolds}

Extremal results and the convergence case of Khintchine-Groshev type theorems give rise to null sets and so the question of their Hausdorff dimension arises naturally. A manifold $M$ which is a $C^{3}$ planar curve with nonvanishing curvature everywhere except on a set with zero Hausdorff dimension is extremal by Schmidt (1964). By extending this and the results of Baker \& Schmidt (1970), R.C. Baker proved that the Hausdorff dimension of $\mathcal{L}_{v}(M)$ is $3 /(v+1)$ for $v \geqslant 2$ (R.C. Baker 1978). It is shown in Dodson, Rynne \& Vickers (1989) that for manifolds $M$ with dimension $m \geqslant 2$ and 2-curved (this specialises to non-zero Gaussian curvature for surfaces in $\mathbb{R}^{3}$ ) everywhere except on a set of Hausdorff dimension at most $m-1$,

$$
\operatorname{dim} \mathcal{L}_{v}(M)=m-1+(n+1) /(v+1)
$$

$\dagger$ A stronger multiplicative version is also proved. These results can be extended to manifolds which can be sliced into suitable curves, such as, for example, analytic manifolds. 
for $v \geqslant n$. Note that this implies that $M$ is extremal and that when $\psi$ is decreasing, this result can be extended to

$$
\mathcal{L}(M ; \psi)=\left\{\mathbf{x} \in M:\|\mathbf{q} \cdot \mathbf{x}\|<\psi(|\mathbf{q}|) \text { for infinitely many } \mathbf{q} \in \mathbb{Z}^{n}\right\} .
$$

Ubiquity has been used to show that the right-hand side of (23) is a general lower bound for the Hausdorff dimension of $\mathcal{L}(M ; \psi)$ when $M$ is a $C^{1}$ extremal manifold in $\mathbb{R}^{n}$ (Dickinson \& Dodson 2000).

Theorem 11 Let $\psi: \mathbb{N} \rightarrow \mathbb{R}^{+}$be decreasing with the lower order $\lambda$. Let $M$ be a $C^{1}$ extremal manifold embedded in $\mathbb{R}^{n}$ and suppose $\lambda \geqslant n$. Then

$$
\operatorname{dim} \mathcal{L}(M ; \psi) \geqslant m-1+(n+1) /(\lambda+1) .
$$

The proof uses the geometry of numbers and Fatou's lemma. The question of the correct upper bound is more difficult but we conjecture that equality holds for nondegenerate manifolds. Some of the results and methods discussed above have been extended to Diophantine approximation of complex and $p$ adic numbers (see Abercrombie 1995; Dickinson, Dodson, \& Jin 1999).

\section{Simultaneous Diophantine approximation on manifolds}

Determining the Hausdorff dimension of the set $\mathcal{S}_{v}(M)$ defined in (20) of simultaneously $v$-approximable points on manifolds can be more difficult than the dual case. When $M$ is the circle $\mathbb{S}^{1}$ and $v>1$, the natural number $q$ is part of the Pythagorean triple $(p, r, q)$. Melnichuk (1979) exploited this to obtain the correct upper bound and (with regular systems) an estimate for the lower bound. In fact using either ubiquity or regular systems, it can be shown that

$$
\operatorname{dim} \mathcal{S}_{v}\left(\mathbb{S}^{1}\right)=1 /(v+1)
$$

for $v>1$ (Dickinson \& Dodson 2001). Exponential sums can be combined with regular systems to obtain estimates for the Hausdorff dimension of $\mathcal{S}_{v}(M)$ for certain manifolds $M$. The argument involves the distribution of rational points near the manifold. Further details on this and other aspects of the theory can be found in Bernik \& Dodson (1999).

Acknowledgment The second author is grateful to ETH, Zürich for its hospitality and to G. Wüstholz for support and the opportunity to give a shorter version of this article at the international meeting to mark Alan Baker's 60th birthday. We are also grateful to H. Dickinson for helpful discussions and for assistance with the preparation of this article. 


\section{References}

Abercrombie, A.G. (1995), The Hausdorff dimension of some exceptional sets of $p$-adic matrices, J. Number Th. 53, 311-341.

Arnol'd, V.I. (1963), Small denominators and problems of stability of motion in classical and celestial mechanics, Usp. Mat. Nauk 18, 91-192; English translation in Russian Math. Surveys, 18 (1963), 85-191.

Arnol'd, V.I. (1983), Geometrical Methods in Ordinary Differential Equations, Springer-Verlag.

Baker, A. (1966), On a theorem of Sprindžuk, Proc. Roy. Soc. Series A 292, 92-104.

Baker, A. (1975), Transcendental Number Theory, Cambridge University Press; second edition (1979).

Baker, A. \& W.M. Schmidt (1970), Diophantine approximation and Hausdorff dimension, Proc. Lond. Math. Soc. 21, 1-11.

Baker, R.C. (1978), Dirichlet's theorem on Diophantine approximation, Math. Proc. Cam. Phil. Soc. 83, 37-59.

Beresnevich, V.V. (1999), On approximation of real numbers by real algebraic numbers, Acta Arith. 90, 97-112.

Beresnevich, V.V. (2000), Application of the concept of regular system of points in metric number theory, Vesti NAN Belarusi. Phys.-Mat. Ser., (1), 35-39.

Beresnevich, V.V. (2001a), On proving Khintchine type theorems for curves, Vesti NAN Belarusi, Phys.-Mat. Ser., in Russian, to appear.

Beresnevich, V.V. (2001b), A generalisation of the Khintchine-Groshev theorem for Diophantine approximation on manifolds, submitted.

Beresnevich, V.V. \& V.I. Bernik (1996), On a metrical theorem of W. Schmidt, Acta Arith. 75, 219-233.

Beresnevich, V.V., V.I. Bernik, H. Dickinson \& M.M. Dodson (1999), The Khintchine-Groshev theorem for planar curves, Proc. Roy. Soc. Lond. A 455, 3053-3063.

Bernik, V.I. (1979), On the exact order of approximation of almost all points on the parabola, Mat. Zametki 26, 657-665.

Bernik, V.I. (1983), An application of Hausdorff dimension in the theory of Diophantine approximation, Acta Arith. 42, 219-253; English translation in American Mathematical Society Translations 140 (1988), 15-44.

Bernik, V.I. (1989), On the exact order of approximation of zero by values of integral polynomials, Acta Arith. 53, 17-28 (in Russian). 
Bernik, V.I. \& V. N. Borbat (1997), Polynomials with coefficients of different modulus and A. Baker's conjecture, Vesti Akad, Navuk Belarus. Ser. Fiz.Mat. Navuk. 3, 5-8 (in Russian).

Bernik, V.I.\& M.M. Dodson (1999), Metric Diophantine Approximation on Manifolds, Cambridge University Press.

Bernik, V.I. \& E.I. Kovalevskaya (1990), Diophantine approximation on $n$ dimensional manifolds in $\mathbb{R}^{2 n}$, Dokl. Bel. 12, 1061-1064.

Bernik, V.I., H. Dickinson \& M.M. Dodson (1998), A Khintchine-type version of Schmidt's theorem for planar curves, Proc. Roy. Soc. Lond. A 454, 179185 .

Bernik, V.I., D.Y. Kleinbock \& G.A. Margulis (1999), Khintchine-type theorems for manifolds: convergence case for standard and multiplicative versions, Preprint 99-092, Bielefeld. Submitted to International Math. Research Notices.

Besicovitch, A.S. (1934), Sets of fractional dimensions (IV): on rational approximation to real numbers, J. Lond. Math. Soc. 9, 126-131.

Bovey, J.D. \& M.M. Dodson (1986), The Hausdorff dimension of systems of linear forms, Acta Arith. 45, 337-358.

Cassels, J.W.S. (1957), An Introduction to Diophantine Approximation, Cambridge University Press.

Dickinson, H. (1993), The Hausdorff dimension of systems of simultaneously small linear forms, Mathematika 40, 367-374.

Dickinson, H. (1994), The Hausdorff dimension of sets arising in Diophantine approximation, Acta Arith 53, 133-140.

Dickinson, H. \& M.M. Dodson (2000), Extremal manifolds and Hausdorff dimension, Duke Math. J. 101, 337-347.

Dickinson, H. \& M.M. Dodson (2001), Diophantine approximation and Hausdorff dimension on the circle, Math. Proc. Cam. Philos. Soc. 130 (2001), 515-522.

Dickinson, H., M.M. Dodson, \& Jin Yuan (1999), Hausdorff dimension and p-adic Diophantine approximation, Indag. Mathem., N.S. 10, 337-347.

Dickinson, H., T. Gramchev, \& M. Yoshino (1995), First order pseudodifferential operators on the torus: normal forms, Diophantine approximation and global hypoellipticity, Ann. Univ. Ferrara, Sez. VII - Sc. Mat. 61, 51-64.

Dickinson, H. \& S.L. Velani (1997), Hausdorff measure and linear forms, J. Reine Angew. Math. 490, 1-36. 
Dodson, M.M. (1992), Hausdorff dimension, lower order and Khintchine's theorem in metric Diophantine approximation, J. Reine Angew. Math. 432 (1992), 69-76.

Dodson, M.M. (1993), Geometric and probabilistic ideas in the metrical theory of Diophantine approximation, Usp. Mat. Nauk 48, 77-106; English translation in Russian Math. Surveys 48 (1993), 73-102.

Dodson, M.M. (1997), A note on metric inhomogeneous Diophantine approximation, J. Austral. Math. Soc. (Series A) 62, 175-185.

Dodson, M.M., B.P. Rynne, \& J.A.G. Vickers (1989), Metric Diophantine approximation and Hausdorff dimension on manifolds, Math. Proc. Cam. Phil. Soc. 105, 547-558.

Dodson, M.M., B.P. Rynne, \& J.A.G. Vickers (1990), Diophantine approximation and a lower bound for Hausdorff dimension, Mathematika 37, 5973.

Dodson, M.M., B.P. Rynne, \& J.A.G. Vickers (1991), Khintchine-type theorems on manifolds, Acta Arith. 57, 115-130.

Dodson, M.M., B.P. Rynne, \& J.A.G. Vickers (1994), The Hausdorff dimension of exceptional sets associated with normal forms, J. Lond. Math. Soc. 49, 614-624.

Harman, G. (1998), Metric Number Theory, Clarendon Press.

Hill, R. \& S.L. Velani (1998), The Jarník-Besicovitch theorem for geometrically finite Kleinian groups, Proc. Lond. Math. Soc. 77, 524-550.

Jarník, V. (1929), Diophantischen Approximationen und Hausdorffsches Mass, Mat. Sbornik 36, 371-382.

Khintchine, A.I. (1924), Einige Sätze über Kettenbruche, mit Anwendungen auf die Theorie der Diophantischen Approximationen, Math. Ann. 92, 115125.

Kleinbock, D.Y. \& G.A. Margulis (1998), Flows on homogeneous spaces and Diophantine approximation on manifolds, Ann. Math. 148 (1998), 339-360.

Koksma, J.F. (1936), Diophantische Approximationen, Springer-Verlag.

Kovalevskaya, E.I. (1978), A geometric property of extremal surfaces, Mat. Zametki 23, 99-101.

Levesley, J. (1998), A general inhomogeneous Jarník-Besicovitch theorem, J. Number Th. 71, 65-80.

Mahler, K. (1932), Über das Mass der Menge aller S-Zahlen, Math. Ann. 106, $131-139$. 
Melián, M.V. \& D. Pestana (1993), Geodesic excursions into cusps in finite volume hyperbolic manifolds, Mich. Math. J. 40, 77-93.

Melnichuk, Y.V. (1979), Diophantine approximations on a circle and Hausdorff dimension, Mat. Zametki 26, 347-354; English translation in Math. Notes 26 (1980), 666-670.

Rynne, B.P. (1992), Regular and ubiquitous systems, and $\mathcal{M}_{\infty}^{s}$-dense sequences, Mathematika 39 (1992), 234-243.

Rynne, B.P. (1998a), Hausdorff dimension and generalised simultaneous Diophantine approximation, Bull. Lond. Math. Soc. 30, 365-376.

Rynne, B.P. (1998b), The Hausdorff dimension of sets arising from Diophantine approximation with a general error function, J. Number Th. 71, 166177.

Schmidt, W.M. (1964), Metrische Sätze über simultane Approximation abhängiger Grössen, Monatsh. Math. 68, 154-166.

Sprindžuk, V.G. (1969), Mahler's Problem in Metric Number Theory, American Mathematical Society (1969), translated by B. Volkmann.

Sprindžuk, V.G. (1979), Metric theory of Diophantine Approximations, Wiley.

Sprindžuk, V.G. (1980), Achievements and problems in Diophantine approximation theory, Usp. Mat. Nauk 35, 3-68; English translation in Russian Math. Surveys, 35, (1980), 1-80.

Vinogradov, A.I. \& G.V. Chudnovsky (1984), The proof of extremality of certain manifolds. In Contributions to the Theory of Transcendental Numbers, G.V. Chudnovsky (ed.), American Mathematical Society, 421-447. 